Arango Benjumea, J. J., Boyero Saavedra, M. R., Carvajal Jaramillo, J. A., Guzmán Monsalve, V. T., Montenegro Velandia, W. y Montoya Monsalve, C. A. (mayo-agosto, 2020). Percepciones entre profesores y estudiantes sobre la didáctica de la contabilidad internacional. Revista Virtual Universidad Católica del Norte, (60), 24-45 https://www.doi.org/10.35575/rvucn.n60a3

\title{
Percepciones entre profesores y estudiantes sobre la didáctica de la contabilidad internacional
}

Perceptions between teachers and students on the teaching of international accounting

\author{
Jhon Jaime Arango Benjumea \\ Magister en Administración \\ Universidad Cooperativa de Colombia \\ Medellín, Colombia \\ jhon.arangob@campusucc.edu.co \\ Orcid: https://orcid.org/0000-0002-4036-3178
}

\section{Javier Alberto Carvajal Jaramillo}

Magister en educación y Tic (e-learning)

Universidad Cooperativa de Colombia

Medellín, Colombia

javier.carvajal@campusucc.edu.co

Orcid: https://orcid.org/0000-0001-7326-5390

\section{Wilson Montenegro Velandia}

Magíster en Administración de Empresas Universidad Cooperativa de Colombia

Medellín, Colombia

wilson.montenegro@campusucc.edu.co

Orcid: https://orcid.org/0000-0003-0846-6692

\section{Martín Ramiro Boyero Saavedra}

Magíster en Educación

Institución Universitaria Escolme

Medellín, Colombia

mrboyeros@escolme.edu.co

Orcid: https://orcid.org/0000-0002-1313-6922

\author{
Verónica Tatiana Guzmán Monsalve \\ Magister en Estética \\ Institución Universitaria Escolme \\ Medellín, Colombia \\ vguzman@escolme.edu.co \\ Orcid: https://orcid.org/0000-0003-3132-4887
}

\section{César Alveiro Montoya Monsalve \\ Doctor en Administración \\ Universidad Cooperativa de Colombia \\ Medellín, Colombia \\ cesar.montoyaag@campusucc.edu.co \\ Orcid: https://orcid.org/0000-0001-7618-4713}

Recibido: 31 de enero de 2019

Evaluado: 25 de marzo de 2020

Aprobado: 04 de mayo de 2020

Tipo de artículo: Investigación Científica y Tecnológica

\section{Resumen}

El objetivo del presente trabajo es describir las percepciones que tienen profesores y estudiantes de los programas de contaduría pública acerca de la didáctica de los cursos de contabilidad internacional que se imparten en las instituciones de educación superior. Para lograr dicho objetivo se realizó una investigación cualitativa, con alcance descriptivo y exploratorio, definiéndose para ello seis categorías didácticas, en términos de educación, objetivos, contenidos, estrategias, recursos y evaluación. Mediante el muestreo por oportunidad, se entrevistaron siete profesores y 22 estudiantes de los programas de contaduría pública de dos universidades de la ciudad de Medellín, Colombia. Se constató que el énfasis en lo conceptual, el desarrollo modular de los contenidos, las clases magistrales, el predominio de diapositivas y la falta de retroalimentación, limitan el aprendizaje y el buen desempeño profesional, requiriéndose más uso de recursos informáticos, más participación del estudiante y mayor validación de la contabilidad internacional, en el contexto local empresarial. Se concluyó que entre profesores y estudiantes existen divergencias respecto de las estrategias didácticas que se utilizan en los cursos de contabilidad internacional, en el ámbito de las instituciones de educación superior. 
Palabras clave: : Contabilidad internacional; Didáctica; Estudiante; Profesor.

\section{Abstract}

The objective of this work is to describe the perceptions that teachers and students have of public accounting programs about the didactics of international accounting courses that are taught in higher education institutions. To achieve this objective, a qualitative research was carried out, with a descriptive and exploratory scope, defining six didactic categories, in terms of education, objectives, content, strategies, resources and evaluation. Using opportunity sampling, seven professors and 22 students from the public accounting programs of two universities in the city of Medellin, Colombia were interviewed. It was found that the emphasis on the conceptual, the modular development of the contents, the master classes, the predominance of slides and the lack of feedback, limit learning and good professional performance, requiring more use of computer resources, more student participation. and further validation of international accounting, in the local business context. It was concluded that there are differences between teachers and students regarding the didactic strategies used in international accounting courses, in the field of higher education institutions.

Keywords: International accounting; Didactic; Student; Teacher.

\section{| Introducción}

La contabilidad internacional ha establecido cambios significativos en la preparación y elaboración de la información financiera por parte de las empresas. En el contexto social y económico de los países latinoamericanos, con predominio de la contabilidad fiscal, es pertinente examinar, en el contexto de los programas de contaduría pública, los mecanismos didácticos que se utilizan para el estudio y aplicación de la contabilidad internacional, la cual está basada más en juicios, principios y estimaciones contables, que en normas de carácter contable y fiscal (Jackquin, De Lange, \& Natoli, 2013; Montenegro-Velandia et al., 2016).

El uso de los principios contables tiene implicaciones epistemológicas (Gómez, 2004) relacionadas con una mayor exigencia en su comprensión y posterior aplicación. La contabilidad internacional, que se construye a partir de los principios contables, exige suministrar información financiera (International Accounting Standard Board -IASB-, 2015) razonable y comprensible para diversos tipos de usuarios, en variados y complejos contextos sociales y económicos (Soto, Salazar y Galvis, 2008); lo anterior, en el entendido de que lo complejo no desdibuja lo simple, sino que lo complementa (Martín-Barbero, 1999), pues lo simple expresa una disposición didáctica, cuyo fundamento pedagógico estriba en la consideración de aprendizajes sencillos que progresivamente se van integrando a otros de mayor complejidad.

En el ámbito del proceso educativo, esto es en el acto de enseñar y de aprender, diversos autores (Bustamante-Salazar, 2015; Churyk, Gross \& Reinstein, 2010; Conrod, 2010; Glover \& Werner, 2015; Gutiérrez, 2008; Hodgdon, Hughes \& Street, 2011; Holtzblatt, Tschakert \& Abu-Khadra, 2012; Jackquin et al., 2013; Larson \& Street, 2011; Mayor-Ríos, Pacheco-Ortiz, Patiño-Vanegas, y Ramos-y-Yovera, 2019; Reyes y Chaparro, 2013; Sangster \& Stoner, 2013; Vysotskaya \& Prokofieva, 2013) coinciden en proponer e integrar estrategias didácticas para la enseñanza y aprendizaje de la contabilidad internacional, que van desde usar metodologías activas, contrastar la 
norma contable nacional e internacional, educar por competencias y usar recursos informáticos, hasta analizar casos reales en empresas para determinar métodos adecuados de reconocimiento y medición de las transacciones económicas, según la dinámica de la actividad local empresarial.

Es de interés investigativo y académico abordar entonces una problemática relacionada con la forma cómo se dictan los cursos de contabilidad internacional en los programas de contaduría pública, con el fin de aportar elementos que contribuyan al mejoramiento didáctico de los mismos. En este orden de ideas, el objetivo de este trabajo es describir las percepciones que tienen los profesores y estudiantes de los programas de contaduría pública acerca de la didáctica de los cursos de contabilidad internacional, que se imparten en las instituciones de educación superior.

La pertinencia del presente trabajo reside, por un lado, en la responsabilidad que tienen las instituciones de educación superior para responder a las necesidades de conocimiento, comprensión y aplicación de la contabilidad internacional, en un contexto empresarial local con altos niveles de informalidad; $y$, por otro lado, en pretender que dicho trabajo sea una base empírica para desarrollar nuevas propuestas didácticas, en estudios posteriores, que estén acorde con las exigencias educativas del contador público actual.

\section{| Referentes teóricos}

Diversos autores plantean la convergencia de variadas estrategias didácticas en el estudio y aplicación de la contabilidad internacional, lo que a su vez sugiere nuevos retos y desafíos para su adecuado manejo en el ámbito educativo. De esta manera, el abordaje educativo de la contabilidad internacional implica articular los planes de estudio con el modelo por competencias (Gutiérrez, 2008; Reyes y Chaparro, 2013), contrastando las normas contables nacionales con las internacionales (Larson \& Street, 2011), con el fin de evidenciar diferencias y similitudes conceptuales y prácticas, las cuales, a partir del uso y adaptación de diversos recursos informáticos (Holtzblatt et al., 2012; Mayor-Ríos et al., 2019), puedan facilitar la enseñanza y aprendizaje de la contabilidad internacional.

Por su parte, las metodologías activas, los ejercicios de investigación (Conrod, 2010; Reyes y Chaparro, 2013; Bustamante-Salazar, 2015), y el uso de casos reales (Churyk et al., 2010), propician en el estudiante de contaduría una mayor participación y un mayor compromiso en su propio proceso de aprendizaje, a la vez que por parte del profesor se exige una permanente cualificación (Sangster \& Stoner, 2013; Vysotskaya \& Prokofieva, 2013), como gestor y orientador esencial en el proceso de enseñanza de las normas de contabilidad internacional.

De otro lado, es esencial el uso de nuevos materiales educativos (Sangster \& Stoner, 2013) y de nuevas estrategias didácticas que permitan al profesor y al estudiante de contaduría una mayor aplicación de los juicios y principios contables, dejando de lado el simple uso de las tradicionales reglas contables (Hodgdon et al., 2011; Jackquin et al., 2013), en el entendido de que la contabilidad internacional va más allá del registro de las transacciones económicas, bajo el modelo de partida doble, a la vez que su abordaje educativo debe estar articulado con el proceso de implementación que se adelanta en las empresas (Glover \& Werner, 2015). 
En consecuencia, el abordaje educativo de la contabilidad internacional sugiere su inclusión en los planes de estudio, así como el uso de estrategias didácticas que van desde las metodologías activas, la adaptación de los recursos informáticos y el estudio comparativo de las normas contables nacionales e internacionales, hasta la investigación contable, de tal manera que se facilite su conocimiento y aplicación en el ámbito empresarial.

\section{| La noción de didáctica}

El término didáctica se refiere a la enseñanza y al aprendizaje. En su concepción clásica, es una parte de la pedagogía que se fundamenta en la teoría conductista de estímulo-respuesta y en la teoría de la instrucción (Davini, 1997), lo que claramente ha limitado la noción de aprendizaje activo y colaborarito por parte del estudiante. En la actualidad, la noción de didáctica ha trascendido la concepción clásica hacia una disciplina que alterna lo teórico, lo práctico, el método y la reflexión crítica (Menin, 2001) de la efectividad del proceso educativo, cuyo interés es el de enseñar y aprender a partir de la validación teórica en contextos reales (Medina y Mata, 2009). En este sentido, el enseñar y el educar es un proceso transformador de la propia realidad.

Por tanto, la didáctica por su condición realista-crítica es una continua revisión y corrección del proceso de enseñanza y aprendizaje, a partir del análisis y estructuración de la realidad educativa (Sevillano, 2005), la cual debe trascender la noción intuitiva de la instrucción, hacia una visión que considere el conocimiento como un trabajo colaborativo, pues la educación es un asunto que se construye con alguien (Litwin, 1997). Esta condición trasciende la noción egoísta de la educación, en el entendido de que el profesor es el dueño del conocimiento, desconociendo la participación del estudiante en su propio proceso de aprendizaje. Así, el estudiante reconfigura y cuestiona lo que el profesor plantea (Cruz-Cruz, 2014), a la vez que valida en su propio contexto lo que percibe.

Por otro lado, la didáctica se ocupa de la instrucción y aplicación de teorías psicológicas de educación (Sevillano, 2005, citando a Khrone, 2002), lo que hace alusión al carácter interdisciplinario de la didáctica, en relación con la psicología, la neurociencia y la lingüística, en tanto que es un ejercicio comunicativo que integra la política, la sociología y la historia, no de manera totalitaria ni unidireccional, pues, aunque se acepta que la didáctica tiene un carácter normativo-educativo en un contexto social cambiante, esta debe tener en cuenta al estudiante como sujeto que interactúa y que se educa (Lévinas, 1991), de manera simultánea en correlación con el avance del proceso educativo.

En este sentido, la didáctica considera que los cambios sociales demandan nuevos modelos didácticos (Sevillano, 2005). Por ende, se supera la noción tripartita de profesor-contenido-estudiante, propia de la concepción tradicional, y se añade la sociedad como un nuevo elemento, lo cual le da un mayor significado al proceso de enseñar y de aprender. Se reconoce entonces la didáctica como una disciplina pedagógica, cuyo carácter pragmático y social se manifiesta en el acto de educar (Camilloni, 1997; Majó y Marqués, 2002). 
En consecuencia, la didáctica hace referencia a estructuras de actividad en las que se concretan los objetivos de la educación, los cuales se manifiestan tanto en el acto de enseñar, por parte del profesor, como en el acto de aprender, por parte del estudiante (Medina y Mata, 2009), en una interacción social y educativa que debe conducir a la creación conjunta de un conocimiento que ayude a transformar la realidad común en la que ambos interactúan. Así, la didáctica ha de tener en cuenta contenidos, objetivos, secuencias, adaptaciones, actores y resultados, a fin de procurar al estudiante información, motivación y auto aprendizaje, y al profesor el juicio sobre cuáles deben ser las acciones para contribuir con dicho propósito (Corredor, Pérez y Arbeláez, 2008), en un recorrido metodológico en el que profesores y estudiantes (Majó y Marqués, 2002; Zabalza, 2007) logren llegar a límites insospechados del conocimiento.

Así las cosas, en la didáctica interactúan la teoría, la práctica, el enseñar y el aprender, como proceso metodológico, secuencial y dialéctico en un contexto social dinámico, en el que la acción transformadora de la educación conduce las abstracciones teóricas hacia una validación práctica, en el continuo intercambio que produce el complejo y dinámico acto de educar.

\section{| El proceso educativo}

El proceso educativo implica que alguien enseña y que alguien aprende (Hernández, 2018; Sevillano, 2005), no de manera aislada, sino de forma interconectada, integral, continua y acumulativa. Las teorías que explican la educación plantean supuestos y premisas que guían la práctica profesoral, y sustentan las respuestas frente a la pregunta de cómo educar; no obstante, la teoría y la práctica poseen un componente normativo que desborda su naturaleza explicativa (Sevillano, 2005).

En este sentido, el acto de educar involucra aspectos observables como la conducta, y no observables como el desarrollo cognitivo (Vásquez, 2014); de tal forma que, en medio de afectaciones de situación e institución (Sevillano, 2005), debe trascender hacia una continua interacción entre profesores y estudiantes, en la que estos últimos deben ser gestores de su propio aprendizaje (Arends, 2007; Majó y Marqués, 2002; Zabalza, 2007), no como una obligación, sino como la condición mediante la cual el individuo y la sociedad se transforman. Sin embargo, es necesario prevenir del riesgo de asumir la educación como una actividad solitaria y discrecional, cercana al arte o destreza individual (Cid, Pérez y Zabalza, 2013), con una clara alusión a la vocación y al personalismo que sobrestima al profesor y subestima al estudiante (Lévinas, 1991; Mounier; 1995).

Al respecto, Arends (2007) defiende la posibilidad de reflexionar el proceso educativo como una condición necesaria para desterrar, cuestionar y destituir prácticas y creencias didácticas que sean limitantes para el aprendizaje del estudiante, y, en consecuencia, puede defenderse la pertinencia de una teoría de la educación que posibilite "un fundamento más racional y científico a la misma práctica de la que surgió" (Sevillano, 2005, p. 27). Inmerso en el acto didáctico, el proceso educativo supone para el profesor la preparación, enseñanza y evaluación de actividades didácticas, y para el estudiante, la autogestión y autoevaluación de su aprendizaje, cuya efectividad está soportada en la interacción, la dimensión espacio-tiempo y en los contenidos, recursos, finalidades y contextos de aplicación (Majó y Marqués, 2002). 
Características como la edad, los conocimientos previos y los estilos e intereses de aprendizaje constituyen el universo de aspectos que median su comprensión y asimilación de lo aprendido, y explica, entre otras cosas, la pertinencia o fracaso de determinadas prácticas didácticas en un determinado contexto, tal como lo señalan Medina y Mata (2009): "no sólo el contexto incide en los otros elementos del proceso, sino que éstos afectan también a la configuración de aquel" (p. 179). El proceso educativo que integra aspectos mentales, técnicos, físicos, éticos, afectivos (Constitución Política de Colombia, 1991), informáticos (Díaz-Barriga, 2013) y sociales (León, 2012), tiene un carácter dinámico y complejo que impide reducirlo a un derrotero de recetas que, intuitiva y simplemente, faciliten el aprendizaje.

Por tanto, el proceso de educar supone la interacción profesor-estudiante, a través de la reafirmación o construcción conjunta de mecanismos didácticos que posibiliten la creación de nuevos conocimientos cuya finalidad es la transformar personas y sociedades.

\section{Metodología}

El objetivo de este trabajo es describir las percepciones que tienen los profesores y estudiantes de los programas de contaduría pública acerca de la didáctica que se utiliza en los cursos de contabilidad internacional, en el ámbito de las instituciones de educación superior.

El enfoque de la investigación es cualitativo con alcance descriptivo y exploratorio, el cual permite un acercamiento al objeto de estudio desde las comprensiones y significaciones que los sujetos construyen acerca de su propia realidad, tal como la experimentaron; aspecto que posibilita realizar un diagnóstico sobre el fenómeno educativo sin imponer los prejuicios del investigador (Hernández, Collado y Baptista, 2014).

Para el desarrollo de la investigación se aplicó un instrumento de evaluación diagnóstica cualitativa sobre la forma como se lleva a cabo el proceso de enseñanza y aprendizaje de la contabilidad internacional, en los programas de contaduría pública de las instituciones de educación superior.

Esta herramienta diagnóstica fue empleada para dos estamentos institucionales: los profesores y estudiantes de los últimos semestres de la carrera de contaduría pública de la Institución Universitaria ESCOLME y de la Universidad Cooperativa de Colombia (UCC), ambas ubicadas en la ciudad de Medellín.

La diferenciación de los estamentos que conforman la unidad de estudio de la presente investigación no pretende desnaturalizar la complejidad misma del proceso educativo, abstrayendo y estudiando por separado lo que en realidad se desenvuelve en una "relación intersubjetiva" entre profesores y estudiantes (Cisterna, 2005, p. 65); solo se pretende distinguir las percepciones que ambos estamentos tienen sobre las estrategias didácticas que se utilizan en los cursos de contabilidad internacional, y con base en ello, generar un abordaje desde diferentes voces que intervienen en el proceso. 
El instrumento diagnóstico fue aplicado a dos grupos focales de estudiantes y profesores de ambas instituciones (dos de cada uno, para un total de cuatro grupos), y corresponde a un cuestionario de preguntas abiertas formuladas a partir de unas categorías apriorísticas o sensibilizadoras del marco teórico, las cuales fueron complementadas con preguntas emergentes que buscaban profundizar aspectos significativos detectados por los investigadores.

Los grupos focales se escogieron por medio de una muestra por oportunidad, a quienes quisieron participar como informantes de la investigación. En la tabla 1 se presenta la muestra que sirvió de fuente primaria para la investigación.

\section{Tabla 1}

\section{Participantes de los grupos focales de la UCC y Escolme}

\begin{tabular}{lcc}
\hline \multicolumn{1}{c}{ Grupo focal } & Estudiantes & Profesores \\
\hline Grupos focales UCC & 12 & 3 \\
Grupos focales ESCOLME & 10 & 4 \\
\hline
\end{tabular}

Nota: Elaboración propia

Tanto los profesores como los estudiantes que participaron en los grupos focales corresponden a los dos últimos semestres del programa de contaduría pública, en ambas instituciones, por considerar que contaban con un conocimiento más avanzado en la contabilidad internacional, que los estudiantes de los primeros semestres.

Las categorías apriorísticas definidas para la evaluación diagnóstica del proceso de formación fueron operativizadas a partir de la propuesta de evaluación contextual de Majó y Marqués (2002), enfocada en revisar la actuación del profesor en lo que respecta a la forma como diseña, desarrolla y evalúa su intervención educativa, considerando los múltiples aspectos que inciden en el logro eficaz del aprendizaje.

De igual forma, se tuvo en cuenta la propuesta de Duque y Ospina (2015), quienes diseñaron una evaluación de los elementos didácticos que se dan en los micro currículos y prácticas docentes del programa de Contaduría Pública de la Universidad de Antioquia.

Los aspectos definidos por tales autores fueron ajustados para enfocar la comprensión del proceso educativo, desde una perspectiva equilibrada entre profesores y estudiantes, de forma que se considere la visión de los propios estudiantes acerca de la forma como han experimentado su proceso de aprendizaje de la contabilidad internacional. En este sentido, la evaluación de la didáctica del proceso educativo alude al carácter integrador de los elementos del acto didáctico. 
Entonces, a partir de los trabajos de Majó y Marqués (2002) y de Duque y Ospina (2015), las categorías didácticas definidas para evaluar el proceso de enseñanza y aprendizaje de la contabilidad internacional en los programas de contaduría pública fueron educación, objetivos, contenidos, estrategias, recursos y evaluación.

Para facilitar la resolución del cuestionario se diseñó por categoría una pregunta abierta con carácter descriptivo, para conocer las percepciones que tienen profesores y estudiantes sobre las estrategias didácticas que se utilizan en los cursos de contabilidad internacional.

El cuestionario que se diseñó y que se aplicó a profesores y estudiantes por categoría se muestra en la Tabla 2.

Tabla 2

Cuestionario por categoría didáctica para profesores y estudiantes

\begin{tabular}{|c|c|c|}
\hline Categoría & Profesor & Estudiante \\
\hline Educación & $\begin{array}{l}\text { ¿Qué actividades despliega para } \\
\text { conocer las características y } \\
\text { necesidades cognitivas, } \\
\text { motivacionales, psicológicas, entre } \\
\text { otras, de los estudiantes antes de llevar } \\
\text { a cabo el proceso de enseñanza? }\end{array}$ & $\begin{array}{l}\text { ¿Qué actividades has experimentado por } \\
\text { parte del profesor para determinar tus } \\
\text { necesidades de aprendizaje, tus } \\
\text { motivaciones y tus conocimientos } \\
\text { previos sobre el tema que vas a } \\
\text { aprender? }\end{array}$ \\
\hline Objetivos & $\begin{array}{l}\text { ¿Cuáles han sido los objetivos de } \\
\text { aprendizaje que ha pretendido lograr } \\
\text { en el proceso de enseñanza de la } \\
\text { contabilidad internacional? }\end{array}$ & $\begin{array}{l}\text { ¿Cuáles han sido los objetivos de } \\
\text { aprendizaje que el profesor ha } \\
\text { pretendido que alcances cuando te ha } \\
\text { enseñado la contabilidad internacional? }\end{array}$ \\
\hline Contenidos & $\begin{array}{l}\text { ¿Cómo realiza la preparación, } \\
\text { selección y secuenciación de los } \\
\text { contenidos que le sirven como insumo } \\
\text { para la enseñanza de la contabilidad } \\
\text { internacional? }\end{array}$ & $\begin{array}{l}\text { ¿Cómo te han parecido los contenidos } \\
\text { (temas, información) ofrecidos por el } \\
\text { profesor como insumo para el } \\
\text { aprendizaje de la contabilidad } \\
\text { internacional? }\end{array}$ \\
\hline Estrategias & $\begin{array}{l}\text { ¿Qué estrategias didácticas suele } \\
\text { utilizar para motivar, orientar y enseñar } \\
\text { la contabilidad internacional? }\end{array}$ & $\begin{array}{l}\text { ¿Qué estrategias didácticas suele utilizar } \\
\text { el profesor para motivar y enseñar la } \\
\text { contabilidad internacional? }\end{array}$ \\
\hline Recursos & $\begin{array}{l}\text { ¿Por medio de que recursos, materiales } \\
\text { y medios se apoya para generar y } \\
\text { facilitar el aprendizaje de la } \\
\text { contabilidad internacional? }\end{array}$ & $\begin{array}{l}\text { ¿Por medio de qué recursos, materiales y } \\
\text { medios se apoya el profesor para generar } \\
\text { y facilitar el aprendizaje de la } \\
\text { contabilidad internacional? }\end{array}$ \\
\hline Evaluación & $\begin{array}{l}\text { ¿Qué actividades suelen desarrollar } \\
\text { como profesor para evaluar el } \\
\text { aprendizaje de la contabilidad } \\
\text { internacional? }\end{array}$ & $\begin{array}{l}\text { ¿Qué actividades suelen desarrollar el } \\
\text { profesor para evaluar el aprendizaje de la } \\
\text { contabilidad internacional? }\end{array}$ \\
\hline
\end{tabular}

Nota. Elaboración propia. 
En la primera fase de la investigación, se realizó una revisión bibliográfica acerca de diversos mecanismos didácticos que son utilizados en los cursos en contabilidad internacional, en diferentes latitudes. En la segunda fase, a partir de los estudios de Duque y Ospina (2015) y Majó y Marqués (2002), se construyó un cuestionario con las siguientes seis categorías didácticas: educación, objetivos, contenido, estrategia, recursos y evaluación.

En la tercera fase, se aplicó el cuestionario a grupos focales conformados por siete profesores, que constituyen el total de los profesores que sirven los cursos en contabilidad internacional en los programas de contaduría pública, de ambas universidades, y a 22 estudiantes de los semestres siete, ocho y nueve que habían asistido a dichos cursos en el segundo semestre de 2016, y que fueron seleccionados por muestreo por oportunidad.

En la cuarta fase, según la propuesta de Osses, Sánchez e Ibáñez (2006), los testimonios capturados en cámaras de video se transcribieron y se agruparon inductivamente en las categorías didácticas antes mencionadas. En la quinta y última fase, se contrastaron, a manera de resultados y discusión, los testimonios de profesores y estudiantes sobre los mecanismos didácticos que son utilizados en los cursos en contabilidad internacional.

La mayor dificultad para la captura y tratamiento de la información se centró en el hecho de que, en el desarrollo de los grupos focales, se podían aflorar opiniones individuales sobre asuntos que no concernían al objeto de investigación. Cuando esta situación se presentaba el moderador del grupo focal hacía un llamado a retomar el hilo de las preguntas formuladas. Otra dificultad se centró en el hecho de personalizar el proceso de enseñanza y aprendizaje de la contabilidad internacional con respecto a la persona del profesor o del estudiante, lo cual tampoco era tema de estudio de la presente investigación. Esto se subsanó orientando, en el transcurso del desarrollo de los grupos focales, a los participantes sobre la intencionalidad de la investigación.

\section{| Resultados}

\section{Estamento profesor}

A continuación, se presenta una síntesis de las respuestas de los profesores a las preguntas planteadas por cada categoría didáctica.

\section{Categoría educación}

Con respecto a esta categoría, los profesores en el primer día de clase, de los cursos en contabilidad internacional, realizan cuestionarios para conocer los conocimientos actuales de los estudiantes; realizan charlas grupales para conocer experiencias académicas, laborales e inquietudes; indagan a los estudiantes sobre su actividad laboral, académica y expectativas de aprendizaje; despiertan en el estudiante el interés y necesidad de que vean el curso como un desarrollo temático estratégico para el desarrollo profesional; instan al estudiante para que adquiera un lenguaje técnico especializado, relacionado con las normas de la contabilidad internacional; dan a conocer al estudiante la importancia de las normas de la contabilidad internacional y lo sensibilizan sobre su conocimiento y aplicación; lo motivan para el conocimiento de las normas de la contabilidad internacional, en complemento con la práctica, la investigación y la experiencia laboral. 


\section{Categoría objetivos}

Con respecto a esta categoría, los profesores tienen como objetivo de aprendizaje alejar a los estudiantes de la parte fiscal y que se interesen más por el aspecto contable de la contabilidad internacional; dar contenidos actualizados, presentando la diferencia entre norma local y la norma internacional; establecer un equilibrio entre la parte conceptual y la parte práctica de la contabilidad internacional, a través de conceptos y ejercicios; buscar el desarrollo de las competencias en los estudiantes, en términos de comprender, analizar y comparar las normas; abordar el conocimiento y aplicación de la contabilidad internacional desde la comparación con las normas locales; generar en el estudiante la sensibilidad por el estudio y aplicación de la contabilidad internacional; promover en el estudiante que la contabilidad internacional es el lenguaje universal de la economía y finanzas de las empresas.

\section{Categoría contenidos}

Con respecto a esta categoría, los profesores expresaron que los cursos de contabilidad internacional están bien estructurados y con una secuencia de aprendizaje adecuada; en el desarrollo de contenidos parten de los conceptos básicos de la contabilidad internacional, para luego abordar conceptos más complejos; se trata de llevar una secuencia en los contenidos propuestos, sin embargo no faltan, en ocasiones, situaciones que se salgan del tema, pues hay estudiantes que tienen experiencia laboral y plantean preguntas relacionadas con un caso de empresa que se esté ocurriendo en el momento; en las primeras asignaturas de la contabilidad internacional se trabaja el marco conceptual, y en las asignaturas posteriores se aborda la aplicación y práctica; es muy importante el proceso de comprensión del conocimiento de la contabilidad internacional, por ello se trabaja en la orientación de tipo contable y financiera, involucrando elementos relacionados con las asignaturas; el estudiante debe buscar otras fuentes de información relacionada con la contabilidad internacional, en complemento a los contenidos vistos en los cursos; es importante capacitar a los profesores en el desarrollo de los contenidos relacionados con la contabilidad internacional.

\section{Categoría estrategias}

Con respecto a esta categoría, los profesores buscan la curiosidad del estudiante mediante el estudio de casos reales, saliendo de lo teórico a la aplicación en las empresas; realizan análisis de las normas de contabilidad internacional, con base en situaciones concretas que tengan las empresas que han implementado las normas; al menos se presentan dos estrategias, una que va relacionada con los estudiantes que no tienen experiencia laboral, lo cuales necesitan aplicación, y los que tienen experiencia laboral que necesitan más conceptualización; la aplicación práctica desde los primeros momentos, y tratarla de entender las normas internacionales desde el punto de vista jurídico, debido a que están redactadas en un lenguaje muy técnico; utilizan talleres permanentes, partiendo de un principio fundamental, que es un marco teórico que puede ser de un 20\% o menos, y un $80 \%$ o más en la parte práctica; todas las unidades deben ser igual de relevantes, generando prácticas e indicándoles a los estudiantes, de una manera técnica, qué significa realmente en una empresa lo que está diciendo la norma internacional como tal; la metodología siempre será clave en el éxito de cualquier proceso educativo o de formación, por tanto, el abordar de manera integral el tema de la contabilidad internacional es muy importante, y en eso se viene trabajando en equipo. 


\section{Categoría recursos}

Con respecto a esta categoría, los profesores utilizan frecuentemente las presentaciones y diapositivas para orientar sus clases; estudian casos prácticos, analizan opiniones de expertos y presentan videos sobre el proceso de implementación de las normas internacionales de contabilidad; también presentan ayudas, con respecto a documentación que se les entrega a los estudiantes para que analicen, e inclusive se parte de empresas multinacionales o grandes empresas, que ya han trabajado la norma, para hacer un comparativo con las empresas locales; lectura de la norma de contabilidad internacional como tal y su aplicación, por medio de talleres o casos prácticos en un escenario real; visitas a sitios web autorizados que publican información fidedigna para el estudio de la contabilidad internacional; los recursos son todas las normas internacionales como tal, iniciando con el marco conceptual, indicándoles y haciéndoles conocer a los estudiantes la parte de cada una de las normas, en relación con los cambios que han tenido; sobre la integralidad, se hace referencia al estudio del marco conceptual, el marco técnico normativo, las interpretaciones, la Ley 1314/2009, y los decretos que se han desprendido de la ley.

\section{Categoría evaluación}

Con respecto a esta categoría, los profesores utilizan la prueba escrita, cuestionario tipo saber pro, y una evaluación cualitativa en el manejo de la norma; además, durante el desarrollo del curso observan el avance del estudiante en el manejo conceptual y práctico de la contabilidad internacional; también el uso de mapas conceptuales para verificar la asimilación de conceptos en los estudiantes; y para verificar la aplicación, talleres prácticos, ya sean individuales o en grupo; uso de la modalidad de taller de aplicación de casos, en cuyo desarrollo se logran evidenciar los avances que tienen los estudiantes sobre el manejo de las normas; evaluación por competencias, en relación con el ser, saber y hacer, y en articulación con el modelo pedagógico de la universidad; énfasis en la estructura de valores que debe desarrollar el estudiante en el aula de clase, de tal manera que esas competencias les permitan interactuar dentro de un contexto laboral específico, con dominio de las normas internacionales; énfasis en el proceso de maduración del estudiante a medida que va teniendo relación con cada una de las normas, y también generando cuestionarios y casos prácticos; énfasis en la formación integral que es la combinación de teorías y prácticas; la formación o evaluación por competencias, tiene que estar acorde a las necesidades o exigencias del mundo laboral y profesional, además permite la actualización.

\section{Estamento estudiante}

A continuación, se presenta una síntesis de las respuestas de los estudiantes a las preguntas planteadas por cada categoría didáctica.

\section{Categoría educación}

Con respecto a esta categoría, los estudiantes expresaron que los profesores dan las bases de la contabilidad internacional, pero que ellos tienen que investigar más; no ven cómo se aplican las normas en una empresa; ha faltado reforzar la parte práctica, para poder entender la norma, porque se lee, pero falta más explicación y más profundización para saber cómo aplicar las normas; la contabilidad internacional depende más de cómo lo va a implementar 
la empresa y no de cómo lo entiendan los estudiantes; algunos profesores hacen una especie de diagnóstico para conocer cuál es el conocimiento que se tiene de la contabilidad internacional; la mayoría de los profesores asumen que el estudiante ya sabe y entran de una al tema, sin indagar conocimientos previos; lo básico es tener conocimientos previos en contabilidad para poder abordar el aprendizaje de la contabilidad internacional; se está en un momento en el que es necesario desaprender y aprender unas cosas nuevas, lo que no es fácil.

\section{Categoría objetivos}

Con respecto a esta categoría, los estudiantes manifestaron que básicamente los profesores dan a conocer el marco conceptual de la contabilidad internacional, pero de la parte práctica muy poco; los profesores tratan de enfocar en que el objetivo de la contabilidad internacional es mostrar información financiera razonable de las empresas; las empresas necesitan implementar las normas de contabilidad internacional para ser más competitivas; la contabilidad internacional es una contabilidad más global, y el sistema contable local es un sistema más tributario que contable; la contabilidad internacional trata de tomar esa información y analizarla financieramente para mostrar la razonabilidad de la empresa, buscando que si se genera un gasto, este se cause en su totalidad el mismo día, para que al momento de tener una visión empresarial se entienda cómo está la empresa al momento y no en un tiempo determinado.

\section{Categoría contenidos}

Con respecto a esta categoría, los estudiantes manifestaron que básicamente es el mismo marco conceptual de la contabilidad internacional, y su estudio se base en lecturas y en análisis de lo que se lee; los contenidos se ven por módulos y algunos tienen una dinámica más práctica, pero eso depende del profesor; algunos estudiantes que trabajan cuentan sus experiencias, y así los contenidos se entienden mejor; los profesores se quedan cortos en el desarrollo de los contenidos por falta de tiempo y quedan temas sueltos que no se pueden profundizar; en algunos casos no se lleva una secuencia de los contenidos de la contabilidad internacional y los temas se presentan desarticulados; la mejor manera de aprender es aplicando conceptos, porque la lectura se queda ahi y simplemente se va olvidando con el pasar del tiempo, pero cuando se escribe es posible profundizar un poco más el conocimiento; el material didáctico que presentan los docentes es el adecuado para cada tema, y se trabaja sobre estados financieros, a partir de la comparación, con base en lecturas y artículos como material de apoyo; los contenidos son muy acertados a la realidad en todos los niveles, pero durante el curso todo lo que se trabaja es muy conceptual, y no se enfatiza en la práctica. Se trabaja mayormente clase magistral y muy poca dinámica.

\section{Categoría estrategias}

Con respecto a esta categoría, los estudiantes plantearon que deben practicar para entender lo que leen, pues el aprender de la contabilidad internacional también depende de ellos y no solo del profesor, es un asunto de parte y parte; en ocasiones, al llegar a la clase, es posible ver estudiantes que ni siquiera se toman el trabajo de buscar el tema; las normas de contabilidad internacional están muy alejadas de lo que se trabaja en el país; la estrategia 
más común es la clase magistral; de igual manera se desarrollan talleres, trabajos de investigación, exposiciones, ejercicios prácticos, encuentros con otras universidades, en donde se realizan debates, de acuerdo a los temas tratados, y trabajos en equipo en el desarrollo de la clase; la motivación para aprender de la contabilidad internacional depende directamente del profesor y de su metodología; hay profesores que tienen mucha experiencias, pero de docencia no tienen nada, no saben transmitir sus conocimientos ni experiencias; una estrategia adecuada por parte del profesor es que debería saber en qué nivel están los estudiantes y cómo los acompañaría para llegar a un nivel superior de conocimiento y aplicación de la contabilidad internacional, pero eso no ocurre.

\section{Categoría recursos}

Con respecto a esta categoría, los estudiantes expresaron que el principal recurso utilizado por los profesores son las diapositivas; algunos profesores utilizan cuestionarios y ejercicios prácticos; algunos profesores utilizan los celulares para plantear preguntas, las cuales deben ser respondidas en el menor tiempo posible, por parte de los estudiantes, y quien responda bien acumula puntos; las mesas redondas son mejores alternativas de aprendizaje, que cuando el profesor utiliza diapositivas y habla y habla, pues cuando se está en un debate, es posible estar pendiente de lo que otros estudiantes dicen para comparar y entender más el tema; diapositivas, textos y cuestionarios, son los recursos más utilizados por los profesores; el principal recurso son las diapositivas y también se basa en las normas de contabilidad internacional, fotocopias y tablero; la mayoría de las clases se empiezan con una explicación del tema, luego se desarrollan talleres en equipos y, al final, se hace una socialización.

\section{Categoría evaluación}

Con respecto a esta categoría, los estudiantes manifestaron que los profesores evalúan por medio de exposiciones y exámenes escritos del marco conceptual; realización de talleres; se evalúa sobre los contenidos y temas que se trabajan en el aula de clase; prácticamente es lo mismo, porque a todos los estudiantes les dan las mismas materias, así venga un profesor nuevo o diferente, es lo mismo; es la misma secuencia, talleres, exámenes, exposición y mesas redondas; nunca han puesto un problema, porque ellos mismos saben que no se tienen las competencias para solucionarlo; evalúan el tema tratado, y como son temas acumulativos pueden colocar cualquier pregunta; los métodos más utilizados son parciales, talleres, investigaciones, exposiciones; los exámenes son individuales y grupales, todos con base en los temas vistos, con preguntas abiertas y ejercicios prácticos; las evaluaciones textuales son muy duras, en tanto no es posible decir, tal cual, lo que se lee, da durísimo, ya que desde la vida laboral se tienen las herramientas, o se consulta y se busca lo necesario; así debería ser la parte del aprendizaje, que se pueda consultar, preguntar y validar, porque a veces se ponen casos que son irreales, eso no sucede en la práctica. 


\section{| Discusión}

Para los profesores la apropiación conceptual de la contabilidad internacional es el punto de partida para entender y manejar las normas, mientras que para los estudiantes es insuficiente cuando no hay aplicación. La explicación conceptual es la característica dominante de la educación tradicional, que considera al profesor como el dador del conocimiento, y al estudiante como el receptor de este. En contraste, mientras que en las empresas ya se está implementando la contabilidad internacional, en el aula se siguen comparando las normas contables nacionales e internacionales, sin trascender hacia su aplicación en contextos reales (Churyk et al., 2010; Hodgdon et al., 2011; Jackquin et al., 2013; Jaramillo \& Osses, 2008; Martín-Barbero, 1999).

La educación tradicional se resiste a lo pragmático y procedimental. A la luz de la teoría crítica, la enseñanza por segmentación en estructuras deterministas carece de sentido, en los momentos actuales, por la complejidad y dinámica de la educación. El acto de educar, esto es, enseñar, aprender, conocer y descubrir, viene de enfrentar la realidad desde la praxis vital. En efecto, el interés del estudiante es validar la teoría en diversidad de situaciones y de intereses implícitos, relacionados con el acto mismo de conocer, aprender, aplicar y ajustar (Habermas, 1968; Osorio, 2008).

En esta misma línea, ostentar el dominio conceptual es propio de la corriente personalista, que exalta lo valioso de los aportes y testimonios puestos al servicio del otro. El profesor que trabaja de acuerdo con su convicción tiene una visión de conjunto limitada para orientar las diferentes conexiones del saber contable. La educación no es conveniente dejarla a merced del parecer o pensamiento individual, pues esta obliga a la reflexión y valoración social, en tanto que el estudiante debe ser reconocido como agente pensante, actuante y gestor de su propio aprendizaje (Dewey y Luzuriaga, 1995; Mounier, 1995).

Sin bien los estudiantes reconocen la importancia de la contabilidad internacional, los objetivos de los cursos no se están alcanzando y las expectativas iniciales no se satisfacen. Entre las razones identificadas se citan que los profesores también están tratando de entender la contabilidad internacional, las clases tienen una orientación magistral, el énfasis se hace en el marco conceptual, falta aplicación de las normas y hay poca participación del estudiante en su aprendizaje. Tal situación es notoria en los países latinoamericanos que centran el aprendizaje en el saber-saber, con limitaciones en el saber-hacer, dejando de lado la visión de conjunto y el carácter ontológico, cognitivo y pragmático de la educación (Dewey y Luzuriaga, 1995; Montenegro-Velandia et al., 2016).

No se desconoce que las normas de contabilidad internacional fueron promulgadas con base en modelos económicos y financieros más desarrollados y complejos que el de los países latinoamericanos, en los que prevalece, en un alto porcentaje, la informalidad empresarial, lo que dificulta la asimilación y aplicación de la contabilidad internacional. En este orden de ideas, es necesario establecer una didáctica para el estudio y aplicación de la contabilidad internacional, la cual debe estar basada en un proceso educativo que favorezca el desarrollo de aquellas competencias que permitan identificar y resolver problemas contables, en concordancia con las características del tejido empresarial local (Cádiz, Astorga, Villanueva y Echenique, 2012; Villamizar-Herrera, Montenegro-Velandia y Salvador-Poveda, 2012). 
En cuanto a los contenidos de los cursos en contabilidad internacional, los profesores argumentan que presentan secuencia y cohesión, mientras que los estudiantes plantean que hay fragmentación y falta de continuidad. Es pertinente reconocer que la estructura modular de los contenidos en los programas de contaduría pública, constituye un obstáculo para el estudio y aplicación de la contabilidad internacional, dado su carácter de norma transversal. Los contenidos evolucionan según el tema, tipo de estudiante y contexto en el cual se apliquen; por tanto, su integración y articulación es tan necesaria como oportuna para que el profesional contable pueda aplicarlos en contextos reales (Garzón y Gómez, 2010; Gvirzt y Palamidessi, 2005; Mateo y Vlachopoulas, 2013; Resweber, 2000; Rueda, Pinzón y Patiño, 2013).

Desde las estrategias didácticas, los cruces temáticos permiten aprovechar la versatilidad de la contabilidad internacional, cuya naturaleza implica la combinación de diversos saberes en materia contable y financiera, generando así un proceso educativo más holístico y menos lineal. Sin embargo, el estudio comparativo entre las normas nacionales e internacionales será efectivo en aquellos estudiantes que cuentan con saberes contables previos, pero no en aquellos que carecen de los mismos (Conrod, 2010; Churyk et al., 2010; Glover \& Werner, 2015; Hodgdon et al., 2011; Jackquin et al., 2013; Larson \& Street, 2011). No obstante, dichos saberes deben ser aprovechados por el profesor para la cualificación de los estudiantes, en una interacción y construcción conjunta del conocimiento, en tanto que el acto didáctico se justifica en la medida en que el abordaje teórico, por precario que sea, se valide en los contextos que le sean aplicables (Cáceres y Lemes, 2006; Majó y Marqués, 2002; Oñate, Palencia \& Pascual, 1987; Saracho, 2005).

De otro lado, el uso recurrente de diapositivas en los cursos en contabilidad internacional entraña aquellos recursos didácticos que soportan la transmisión lineal y unidireccional de conceptos del profesor al estudiante, dejando en segundo lugar el sentido práctico de las normas, el cual está relacionado con el desarrollo de competencias para preparar y presentar información que refleje la realidad financiera de las empresas. Existen, por tanto, deficiencias en la educación del profesional contable con respecto al conocimiento y aplicación de las normas de contabilidad internacional, las cuales, de acuerdo con la estructura de los negocios, deben reflejar una información financiera que sea esencial para tomar decisiones (Soto et al., 2008), desde el punto de vista de las inversiones, financiación y distribución de excedentes.

Con respecto a otros recursos didácticos, los profesores manifiestan que algunas aulas no cuentan con las condiciones básicas necesarias para una adecuada educación; entre los factores que mencionan están las altas temperaturas, el ruido y la iluminación deficiente. Por su parte, los estudiantes manifiestan que las jornadas académicas son muy extensas y en horarios inapropiados, como las horas a la mitad del día; estos factores, de una u otra forma, afectan la concentración y atención del estudiante, a la vez que generan desgaste y cansancio por parte del profesor. Para algunos estudiantes es evidente que las universidades deben pensar más en la comodidad física de los estudiantes para un adecuado proceso de educación.

En relación con la evaluación, profesores y estudiantes coinciden en que las técnicas más 
utilizadas son talleres, exámenes, exposiciones y mesas redondas. Más que técnicas, la evaluación implica la retroalimentación por parte del profesor y la autoevaluación por parte del estudiante, las cuales se deben conjugar para valorar los avances y resultados del aprendizaje; la evaluación así supone la manifestación más visible del acto de educar y aprender (García, 2015; Majó y Marqués, 2002; Ministerio de Educación Nacional - MEN, 2016). Sin embargo, esto no se logra cuando dicho proceso está sesgado por el dominio conceptual del profesor, que coloca al estudiante en una posición de pasividad frente al cuestionamiento crítico que debe conducir a la creación conjunta de conocimientos (García, 2015; Dewey y Luzuriaga, 1995; Mounier, 1995).

En efecto, un adecuado proceso de evaluación en contabilidad internacional permite que el estudiante tenga participación en la valoración de su aprendizaje, con el complemento de la valoración del profesor que debe asumir el rol de mentor y orientador, cada vez con menor protagonismo, notoriedad y dominio soberano del saber contable. El desafío es, por tanto, procurar que en los cursos en contabilidad internacional se impartan conocimientos y se promueva el desarrollo de competencias para su posterior aplicación en el mundo real (García, 2015; Majó y Marqués, 2002).

Finalmente, es pertinente plantear ajustes a la didáctica de los cursos en contabilidad internacional, especialmente en el componente de validación conceptual en contextos reales. En tal sentido, se plantea como modelo didáctico aquel que con la orientación del profesor propicie el autoaprendizaje de los estudiantes, utilizando metodologías activas, cuyo sentido práctico supone el uso del marco conceptual de la contabilidad internacional en escenarios reales y virtuales, de forma tal que su comprensión resulte significativa y necesaria para la praxis del profesional contable.

Con base en lo anterior, en la figura 1 se plantea un modelo didáctico que considera a la contabilidad internacional, al profesor, al estudiante, al contexto y a la evaluación como los ejes centrales del proceso educativo en los cursos en contabilidad internacional, de los programas de contaduría pública en las instituciones universitarias.

\begin{tabular}{|c|c|c|c|c|}
\hline IfRs & PROFESOR & ESTUDIANTE & CONTEXTO & EVALUACIÓN \\
\hline \multirow{2}{*}{ Marco conceptual } & Instruccion & Asimilación & \multirow{2}{*}{ Aplicación } & \multirow{2}{*}{$\begin{array}{l}\text { Retroalimentacion } \\
\text { Autoevaluación }\end{array}$} \\
\hline & \multicolumn{2}{|c|}{$\begin{array}{l}\text { Educación, objetivos, contenidos, estrategias, } \\
\text { recursos }\end{array}$} & & \\
\hline \multirow{2}{*}{$\begin{array}{l}\text { Juicios, principios, } \\
\text { estimaciones }\end{array}$} & Meto & activas & \multirow{2}{*}{$\begin{array}{l}\text { Universidad } \\
\text { Empresa }\end{array}$} & \multirow{2}{*}{$\begin{array}{l}\text { Aprendizaje } \\
\text { colaborativo }\end{array}$} \\
\hline & \multicolumn{2}{|c|}{$\begin{array}{c}\text { Clases invertidas, estudio de casos, visitas } \\
\text { empresariales, aprendizaje basado en } \\
\text { problemas }\end{array}$} & & \\
\hline
\end{tabular}

Figura 1 . Modelo didáctico de la contabilidad internacional. Fuente: resultados y discusión de investigación. 
De acuerdo con la figura 1, tanto la secuencialidad como la transversalidad son esenciales en el proceso educativo, en los cursos en contabilidad internacional. El aspecto fundamental del marco conceptual es el manejo de los juicios, principios y estimaciones contables, para reconocer y medir de forma adecuada las transacciones económicas. El profesor instruye y el estudiante, de manera activa, asimila conceptos soportados en la educación, objetivos, contenidos, estrategias y recursos, cuyo aprendizaje debe ser el resultado natural del uso de diversas metodologías activas. Lo anterior, debe conducir a la aplicación de los conceptos mediante ejercicios prácticos, propios del modelo académico, así como con la validación en las empresas nacionales o internacionales que han llevado a cabo procesos de implementación de la contabilidad internacional. Por último, como es de esperar, debe ocurrir la evaluación en doble vía, esto es la retroalimentación del profesor y la autoevaluación del estudiante, en consonancia con el enfoque del aprendizaje colaborativo, para realizar los ajustes, adaptaciones y correcciones que sean necesarios al proceso educativo.

\section{| Conclusiones}

El objetivo del presente trabajo fue describir las percepciones que tienen los profesores y estudiantes de los programas de contaduría pública, acerca de la didáctica que se utiliza en el desarrollo de los cursos de contabilidad internacional y que son impartidos en las instituciones de educación superior.

Al respecto, se pudo establecer que entre profesores y estudiantes se presentan divergencias significativas, especialmente en la estrategia didáctica que hace referencia al equilibrio y relación que debería existir entre la teoría y práctica. Mientras que, para los profesores, en las actividades que se desarrollan en los cursos de contabilidad internacional, existe un equilibrio y una buena relación entre la teoría y la práctica, para los estudiantes hay un exceso en lo conceptual con grandes limitaciones en la práctica y aplicación de los temas tratados en dichos cursos.

Como complemento a lo anterior, los profesores consideran que el desarrollo de talleres, la realización de ejercicios y el estudio de casos constituyen en esencia la aplicación de las normas de la contabilidad internacional. Por su parte, los estudiantes, consideran que la práctica debe ser en contextos reales o en contextos que simulen la realidad empresarial y en total correspondencia con la implementación de las normas según las características de cada empresa en particular.

Por esta razón, se presume que el énfasis en lo conceptual, con restricciones de aplicación en contextos reales, limita el desempeño del profesional contable en el ámbito local, nacional, regional e incluso internacional, dada la condición de globalidad con la cual fueron concebidas dichas normas. Esto es debido a que reflejar la realidad financiera de las empresas se asume como una consecuencia lógica de la comprensión y aplicación del marco conceptual de la contabilidad internacional, sin la debida validación del efecto contable y financiero que tiene su implementación en los contextos reales.

En similar sentido, el desarrollo modular de los contenidos fracciona el aprendizaje y 
desarticula el conocimiento contable en lo conceptual y técnico. De manera similar, la clase magistral, el predominio de diapositivas con poco uso de recursos informáticos y la escasa o nula retroalimentación por parte del profesor, limitan la participación del estudiante como coautor y coevaluador de su propio proceso de aprendizaje. A esto se le adiciona que, si bien el estudio comparativo de las normas contables locales e internacionales es una estrategia didáctica adecuada, es necesario migrar solo al estudio y aplicación de las normas de contabilidad internacional.

Al respecto, los métodos de estudio y de aplicación de la contabilidad internacional, basados en enfoques lineales y determinísticos, cercanos al conductismo subyacente, poseedor de la verdad, que descompone el conocimiento en unidades de información y bloques de actividades, no permiten al estudiante el desarrollo autónomo de su propio aprendizaje. Esto se enfatiza en que el profesor de forma unidireccional se limita a la transmisión de conceptos desde su propia convicción. Esta condición, no reconoce al estudiante como sujeto creador de saberes conjuntos. Pues si la realidad es aquello que se opone a la fantasía, la sensibilidad por el otro será el polo a tierra que orienta un constructo sano y firme, un espacio para pensar en lo humano y romper con la ontología de poseer la realidad absoluta.

De esta manera, las percepciones que ambos estamentos, profesores y estudiantes, tienen sobre las estrategias didácticas que son utilizadas en el desarrollo de los cursos de contabilidad internacional, entran en conflicto y, unos y otros, defienden sus posturas y no se vislumbra una conciliación con respecto a un mejor aprovechamiento práctico del marco conceptual de las normas. No se discute la importancia que tiene la implementación de las normas en contextos reales, pero dicha implementación carecería de toda validez si no obedece a un estudio, análisis y comprensión adecuada de la teoría que la precede.

La mayor limitación de la investigación estuvo determinada por el número reducido de profesores y estudiantes que conformaron los grupos focales, debido a la poca participación que estos tienen en los cursos de contabilidad internacional. No obstante, los autores consideran que se logró abordar el fenómeno de investigación, debido a que las percepciones entre profesores y estudiantes, sobre la didáctica en los cursos en contabilidad internacional, permitió evidenciar que hay desarticulación entre la enseñanza que se imparte y el aprendizaje que se recibe.

El presente trabajo es sólo una parte de un estudio no terminado, pero en continua indagación que propende porque se aborden a futuro aspectos como la inclusión transversal de la contabilidad internacional en los planes de estudio, una mayor participación de los estudiantes en su autoaprendizaje, la inclusión de escenarios prácticos y entornos virtuales para que profesores y estudiantes recreen en tiempo sincrónico y asincrónico la comprensión y aplicación de la contabilidad internacional; así mismo, estudios con los que se pueda identificar los factores que son determinantes para el fortalecimiento de la relación universidad-empresa en el campo de la comprensión e implementación de las normas de contabilidad internacional. 


\section{Referencias}

Arends, R. (2007). Aprender a educar. México: McGraw-Hill.

Asamblea Nacional Constituyente. (1991). Constitución Política De Colombia.

Bustamante-Salazar, A. M. (2015). Costeo basado en actividades -ABC: revisión de literatura. Revista CEA, 1(1), 109-119. https://doi.org/10.22430/24223182.72

Cáceres, A. G. y Lemes A. B. (2006). Enfoque de la interdisciplinaridad y transdisciplinaridad en la educación de la economía política. Revista Iberoamericana de Educación, 41(1), 1-11.

Cádiz, J., Astorga, M. L., Villanueva, O. L. y Echenique, M. J. (2012). ¿Profesores competentes o humanizadores? Educación y Educadores, 15(3), 535-546. Recuperado de http://educacionyeducadores.unisabana.edu.co/index.php/eye/article/view/2453

Camilloni, A. W. (1997). De herencias, deudas y legados: una introducción a las corrientes actuales de la didáctica. En A. W. Camilloni, M. C. Davini, G. Edelstein, E. Litwin, M. Souto y S. Barco (Eds.), Corrientes didácticas contemporáneas (pp. 17-39). Buenos Aires, Argentina: Paidós. Recuperado de https://www.academia.edu/6245374/Camilloni_Davini_Corrientes_didacticas_contemporaneas_Cap_1_y_2

Churyk, N. T., Gross, M. G. \& Reinstein, A. (2010). Raleigh building products: A teaching case that highlights the differences between IFRS and US GAAP. Journal of accounting education, (28), 128-137.

Cid, A. S., Pérez, A. A. y Zabalza, M. B. (2013). Las prácticas de enseñanza realizadas/observadas de los mejores profesores de la Universidad de Vigo. Educación XXI, 16(2), 264-296.

Cisterna, F. (2005). Categorización y triangulación como procesos de validación del conocimiento en investigación cualitativa. Theoria, 14(1), 61-71. Recuperado de https://www.redalyc.org/articulo.oa?id=29900107

Conrod, J. E. (2010). IFRS within the Multi-GAAP Canadian Reality: A Teaching and Learning Imperative for Intermediate Financial Accounting. Accounting perspectives / perspectives comptables, 9(1), 1-13.

Corredor M. V., Pérez M. I. y Arbeláez, R. (diciembre, 2008). Estrategias de enseñanza y aprendizaje. Docencia Universitaria, 9, 155-159

Cruz-Cruz, E. Y. (2014). La didáctica es humanista. Perfiles Educativos, 36(145), 213-220. https://doi.org/10.1016/S0185-2698(14)70647-6

Davini, M. C. (1997). Conflictos en la evolución didáctica: la demarcación entre la didáctica general y las didácticas especiales. En A. W. Camilloni, M. C. Davini, G. Edelstein, E. Litwin, M. Souto y S. Barco (Eds.), Corrientes didácticas contemporáneas (pp. 41-73). Buenos Aires, Argentina: Paidós. https://www.academia.edu/6245374/Camilloni_Davini_Corrientes_didacticas_contemporaneas_Cap_1_y_2 
Dewey, J. y Luzuriaga, L. (1995). Democracia y educación. Madrid, España: Morata.

Díaz-Barriga, A. (2013). TIC en el trabajo del aula. Impacto en la planeación didáctica. Revista Iberoamericana de Educación Superior, 4(10), 3-21. https://doi.org/10.1016/S2007-2872(13)71921-8

Duque, M. I. y Ospina, C. M. (2015). Elementos para discusión sobre el estado actual de la propuesta curricular del programa de contaduría pública de la Universidad de Antioquia. Cuadernos de Contabilidad, 16(41), 355-394. http://dx.doi.org/10.11144/Javeriana.cc16-41.edea

García, E. J. (2015). La evaluación del aprendizaje: de la retroalimentación a la autorregulación. El papel de las tecnologías. Relieve, 21, 1-25.

Garzón, O. y Gómez, J. P. (julio-diciembre, 2010). Diálogos entre la articulación curricular y la formación investigativa. Revista científica Guillermo de Ockham, 8(2), 85-99.

Glover, H. \& Werner, E. (13 de enero de 2015). Teaching IFRS: Options for Instructors. Advances in Accounting Education: Teaching and Curriculum, 113-131. Recuperado de http://www.emeraldinsight.com/doi/abs/10.1108/S1085-462220150000016006

Gómez, M. V. (2004). Una evaluación del enfoque de las Normas Internacionales de Información Financiera (NIIF) desde la teoría de la contabilidad y el control. Innovar Revista de Ciencias Administrativas y Sociales (24), 112-131. Recuperado de https://revistas.unal.edu.co/index.php/innovar/article/view/25192/25680

Gutiérrez, C. (2008). Propuesta de conocimientos y competencias requeridas por el profesional contable en Chile derivado de la adopción de las NIIF. Capic Review, 6, 47-60. Recuperado de http://www.capic.cl/wp-content/uploads/2015/10/vol6art4.pdf

Gvirzt, S. y Palamidessi, M. (2005). El ABC de la tarea profesor. Currículum y educación. Buenos Aires, Argentina: Aiqué.

Habermas, J. (1968). Conocimiento e interés. Madrid, España: Taurus.

Hernández, R., Collado, C. y Baptista, P. (2014). Metodología de la investigación. México: McGraw-Hill.

Hernández, R. M. (2018). La estrategia didáctica frente a los estilos de aprendizaje en la educación superior. Educación Médica, 19(2). https://doi.org/10.1016/j.edumed.2017.10.034

Hodgdon, C., Hughes, S. B. \& Street, D. L. (August, 2011). Framework-based teaching of IFRS judgements. Accounting Education: An International journal, 20(4), 415-439.

Holtzblatt, M., Tschakert, N. \& Abu-Khadra, H. (2012). Teaching IFRS with Online Videos and Webcasts. Advances in Accounting Education: Teaching and Curriculum Innovations, 13, 425-444. 
International Accounting Standard Board - IASB. (2015). Norma Internacional de Información Financiera para Pequeñas y Medianas Entidades (IFRS para las pymes). Recuperado de https://www.nicniif.org/home/descargar-documento/2426-norma-internacional-de-informacion-financiera-para-pymes_2016.html

Jackquin, B., De Lange, P. A. \& Natoli, R. (2013). Transitioning to IFRS in australian classrooms: Impact on teaching approaches. Issues in Accountig Education, 28(2), 263-275.

Jaramillo, S. \& Osses, S. (2008). Metacognición: un camino para aprender a aprender. Estudios pedagógicos, 34(1), 187-197.

Larson, R. K. \& Street, D. (August, 2011). FRS Teaching Resources: Available and Rapidly Growing. Accounting Education: An International Journal, 20(4), 317-338.

León, A. R. (2012). Los fines de la educación. Orbis, 8(23), 4-50. Recuperado de http://www.redalyc.org/articulo.oa?id=70925416001

Lévinas, E. (1991). Ética e infinito. Madrid, Colombia: La balsa de la medusa.

Litwin, E. (1997). El campo de la didáctica: la búsqueda de una nueva agenda. En A. W. Camilloni, M. C. Davini, G. Edelstein, E. Litwin, M. Souto y S. Barco (Eds.), Corrientes didácticas contemporáneas (pp. 91-116). Buenos Aires, Argentina: Paidós. Recuperado de https://docer.com.ar/doc/xes855

Majó, J. y Marqués, P. (2002). La revolución educativa en la era internet. Barcelona, España: CissPraxis.

Martín-Barbero, J. (1999). La educación en el ecosistema educativo. Comunicar, 13, 13-21.

Mateo, J. y Vlachopoulas, D. (2013). Evaluación en la universidad para el contexto de un nuevo paradigma para la educación superior. Educación XXI, 16(2),183-208.

Mayor-Ríos, J. A., Pacheco-Ortiz, D. M., Patiño-Vanegas, J. C., y Ramos-y-Yovera, S. E. (2019). Análisis de la integración del Big Data en los programas de contaduría pública en universidades acreditadas en Colombia. Revista CEA, 5(9), 53-76. https://doi.org/10.22430/24223182.1256

Medina, A. y Mata, F. (2009). Didáctica general. Madrid, España: Pearson educación.

Menin, O. (2001). La didáctica. En Autor, Pedagogía y Universidad. Currículum, didáctica y evaluación (pp. 29-37). Argentina: HomoSapiens.

Ministerio de Educación Nacional -MEN-. (2016). Currículo. Recuperado de http://www.mineducacion.gov.co/1621/article-79413.html

Montenegro-Velandia, W., Cano-Arroyave, A. M., Toro-Jaramillo, I. D., Arango-Benjumea, J. J., Montoya-Agudelo, C. A., Vahos-Correa, J. E. ... y Coronado-Ríos, B. (2016). Estrategias y metodologías didácticas, una mirada desde su aplicación en los programas de Administración. Educación y Educadores, 19(2), 205-220. http://doi.org/10.5294/edu.2016.19.2.2 
Mounier, E. (1995). Le Personnalisme (16 ed.). Paris: Presses Universitaires de France.

Oñate, C., Palencia, A. \& Pascual, M. A. (1987). Interdisciplinarity: A fundamental frame for the future in the Polytechnic University of Madrid. En SEFI Annual Conference. Conferencia llevada a cabo en Helsinki, Finlandia.

Osorio, S. N. (2008). Bioética y pensamiento complejo I: un puente en construcción. Bogotá, Colombia: Universidad Militar Nueva Granada.

Osses, S., Sánchez, I. e Ibáñez, F. M. (2006). Investigación cualitativa en la educación. Hacia la generación de teoría a través del proceso analítico. Estudios Pedagógicos, 32(1), 119-133. Recuperado de http://ref.scielo.org/zft8bq

Resweber, J. P. (2000). El método interdisciplinario (María Elvira Rodríguez-Luna, trad.). Bogotá, Colombia: Universidad Distrital Francisco José de Caldas

Reyes, N. y Chaparro, F. (2013). Metodologías activas para la enseñanza de las normas internacionales de información financiera en un ambiente virtual de aprendizaje. Cuadernos de Contabilidad, 14(36), 1147-1182. Recuperado de http://www.scielo.org.co/pdf/cuco/v14nspe36/v14nspe36a11.pdf

Rueda, G., Pinzón, J. E. y Patiño, R. A. (2013). Los currículos de los programas académicos de contaduría pública, tras la enseñanza de lo internacional y la globalización en la contabilidad: necesidades de ajuste más allá de respuestas técnicas. Cuadernos de Contabilidad, 14(35), 639-667.

Sangster, A. \& Stoner, G. N. (2013). Teaching IFRS in the U.K.: Contrasting Experiences from Both Sides of the University Divide. Issues in Accounting Education, 28(2), 291-307.

Saracho, M. J. (2005). Un modelo general de gestión por competencias. Modelos y metodologías para la identificación y construcción de competencias. Santiago, Chile: RIL Editores.

Sevillano, M. (2005). Didáctica en el siglo XXI: ejes en el aprendizaje y enseñanza de calidad. Madrid, España: McGraw-Hill.

Soto, E. M., Salazar, C. A. y Galvis, O. de J. M. (2008). Fundamentos teóricos del modelo contable común para las pymes de américa latina: una alternativa a la regulación contable internacional IASB. Estudios Gerenciales, 24(107), 59-86.

Vásquez, A. (2014). Enseñanza, aprendizaje y evaluación en la formación de docentes en educación CTS en el contexto del siglo XXI. Uni-pluri/versidad, 14(2), 37-49.

Villamizar-Herrera, L. N., Montenegro-Velandia, W. y Salvador-Poveda, J. (2012). Revisión teórica sobre la enseñanza y aprendizaje de las matemáticas. Revista Virtual Universidad Católica Del Norte (35), 254-287. http://revistavirtual.ucn.edu.co/index.php/RevistaUCN/article/view/361

Vysotskaya, A. \& Prokofieva, M. (2013). The difficulties of teaching IFRS in Russia. Issues in Accounting Education, 28(2), 309-319.

Zabalza, M. A. (2007). Competencias docentes del profesorado universitario. Calidad y desarrollo profesional. Madrid, España: Narcea. 